\title{
Laparoscopic vs Open Excision of Urachal Remnants in Children
}

\section{Journal of Surgery}

\author{
Alia Whitehead, L. Grier Arthur and Rajeev Prasad* \\ Department of Pediatric General, Thoracic, and Minimally Invasive \\ Surgery, St. Christopher's Hospital for Children/ Drexel University \\ College of Medicine, Philadelphia, PA, USA \\ *Address for Correspondence \\ Rajeev Prasad, MD, Department of Pediatric General, Thoracic, and \\ Minimally Invasive Surgery, St. Christopher's Hospital for Children/ \\ Drexel University College of Medicine, 3601 A Street, Philadelphia, \\ PA 19134, USA, Tel: 215-427-5446; Fax: 215-762-4616; E-mail: \\ rajeev.prasad@tenethealth.com \\ Copyright: ( $) 2015$ Whitehead A, et al. This is an open access article \\ distributed under the Creative Commons Attribution License, which \\ permits unrestricted use, distribution, and reproduction in any medium, \\ provided the original work is properly cited. \\ Submission: 22 November 2014 \\ Accepted: 29 December 2014 \\ Published: 02 January 2015 \\ Reviewed \& Approved by: Dr. Gazi B Zibari, Professor of Surgery, \\ Louisiana State University Health Sciences Center, USA
}

\section{Materials and Methods}

Results: There were 4 patients in the laparoscopic group and 10 in the open excision group. There was no difference in age at surgery between the two groups. There was no difference in operative time (laparoscopic: $66.75 \mathrm{~min}$ vs open: $57.2 \mathrm{~min}$, p-value 0.50 ). There was a trend toward increased cost and longer post-operative length of stay in the laparosc opic group ( $\$ 60594.05$ vs $\$ 40454.83 ; 43.25$ hours vs 9 hours) but neither was statistically significant ( $p$-values 0.11 and 0.058 , respectively). There were no complications in either group.

Disc ussion: La pa rosc opic excision of ura chal remnantsisa sa fe and effective altemative to open excision. In addition, the laparoscopic approach allows for excellent visua lization of the entire urachal tract. Although patients undergoing laparoscopic excision had potentially longer hospital stays, this did not necessa rily translate into higher costs.

\section{Introduction}

A urachal remnant forms when the allantois, the embryologic connection between the bladder and the umbilicus, fails to completely obliterate. This represents a spectrum of disease that includes urachal cyst, urachal sinus, patent urachus, and vesicourachal diverticulum [1]. The type of anomaly predicts the presenting symptoms. A patent urachus will generally present with clear drainage from the umbilicus while urachal cysts will generally present with infection [1-4]. Urachal anomalies are also associated with hypospadias, meatal stenosis, vesicoureteral reflux, and ureteropelvic obstruction [5]. They can also undergo malignant degeneration; however, this accounts for less than $1 \%$ of bladder neoplasms [6].

Given the risk of recurrent infection and malignancy, it is generally accepted that urachal remnants should be excised once discovered $[1,3-6]$. The procedure has historically been performed using an open technique. The laparoscopic approach was introduced to the pediatric population in 1995 [2] and has been deemed safe and effective $[2,5,7]$. While there are several case reports of laparoscopic excision of urachal remnants $[2,7]$, there are few studies comparing open and laparoscopic excision in adults $[8,9]$ and none in children. Unfortunately, urachal anomalies are rare enough, affecting only about 1 in 5000 live births [2], that a randomized, prospective study would be difficult to conduct.

The purpose of this study is to perform a retrospective comparison of the open and laparoscopic techniques at our institution.
After approval by the Institutional Review Board (protocol \# 1303001965), we conducted a retrospective chart review of all patients undergoing open or laparoscopic excision of a urachal remnant by one of five different board certified pediatric surgeons at St. Christopher's Hospital for Children, a free-standing children's hospital, from June 2007 to January 2013. Chosen operative technique was based on surgeon preference. We collected the following data for each patient: age, length of stay, gender, presentation, type of procedure performed, operative time, total hospital expenses, and any complications. Means and standard deviations were calculated using Microsoft Excel (Microsoft, Redmond, WA). P-values and standard deviation were calculated using GraphPad InStat 3 (GraphPad Software, San Diego, CA).

We performed the laparoscopic approach in a similar fashion to previously described techniques. We utilized three ports: one at the umbilicus and two along the left side of the abdomen. Dissection of the urachal remnant is carried out using electrocautery and the ligation is performed using an endoloop. The umbilicus was not excised.

\section{Results}

There were 4 patients in the laparoscopic group and 10 in the open excision group. Table 1 shows the comparisons between the two groups. There was no difference in age at surgery between the two groups ( 4.38 vs 3.58 years, p-value 0.82 ). Five patients presented with infection. Four presented with drainage from the umbilicus. Two patients had chronic abdominal pain, and another two had umbilical granulomas. The final patient was diagnosed prenatally. Tables 2 and 3 give details on each patient from both groups. There was no difference in operative time (laparoscopic: $66.75 \mathrm{~min}$ vs open: $57.2 \mathrm{~min}, \mathrm{p}$-value 0.50 ). There was a trend toward increased cost and longer post-operative length of stay in the laparoscopic group ( $\$ 60594.05$ vs $\$ 40454.83 ; 43.25$ hours vs 9 hours) but neither was statistically significant (p-values 0.11 and 0.058 , respectively). There were no complications in either group.

\section{Discussion}

Laparoscopic excision of urachal remnants is a safe and effective 
ISSN: 2332-4139

Table 1: Comparison of open versus laparoscopic groups.

\begin{tabular}{|l|c|c|}
\hline & Open (n=10) & Laparoscopic ( $\mathbf{n = 4 )}$ \\
\hline Age (years) & 3.58 & 4.38 \\
\hline Operative Time (minutes) & 57.2 & 66.75 \\
\hline Cost (USD) & $40,454.83$ & 0.82 \\
\hline Length of Stay (hours) & 9 & 0.50 \\
\hline Complications & 0 & 43.5 \\
\hline
\end{tabular}

Table 2: Details for patients undergoing open procedure.

\begin{tabular}{|c|c|c|c|c|c|}
\hline Patient & Age (years) & Gender & $\begin{array}{c}\text { Length of Stay } \\
\text { (hours) }\end{array}$ & Weight (kg) & Presentation \\
\hline 1 & 6 & M & 0 & 22.7 & infection \\
\hline 2 & 0.55 & F & 12 & 7 & infection \\
\hline 3 & 0.58 & M & 0 & 9 & umbilical drainage \\
\hline 4 & 0.33 & M & 0 & 7.2 & umbilical granuloma \\
\hline 5 & 0.02 & F & pre-natal & clear umbilical drainage \\
\hline 6 & 0.25 & M & 0 & 3.29 & clear umbilical drainage \\
\hline 7 & 11 & F & 0 & 5.5 & clear umbilical drainage \\
\hline 8 & 2 & M & 0 & 40 & 38 \\
\hline 9 & 15 & M & 0 & 18 & umbilical pain \\
\hline
\end{tabular}

Table 3: Details for patients undergoing laparoscopic procedure.

\begin{tabular}{|c|c|c|c|c|c|c|}
\hline Patient & Age (years) & Gender & Length of Stay (hours) & Weight (kg) & Presentation \\
\hline 1 & 0.33 & M & 29 & 7.35 & Operative Time (minutes) \\
\hline 2 & 1 & M & 72 & 12 & abdominal pain \\
\hline 3 & 16 & M & 0 & 64.6 & infection \\
\hline 4 & 0.17 & M & 72 & 4.2 & infection \\
\hline
\end{tabular}

alternative to open excision. In addition, the laparoscopic approach allows for excellent visualization of the entire urachal tract and bladder. This may allow for a more complete excision and a lower risk for future malignancy. Some argue that the lateral laparoscopic approach can even be utilized during acute umbilical infection, while the open approach during an acute infection is associated with significant morbidity $[5,10]$. As with all laparoscopic procedures, there is the potential for more rapid recovery, improved cosmesis, and less postoperative pain. Although these have not been specifically addressed in our study, Masuko et al. felt that the laparoscopic approach offered good cosmetic outcomes [11].

Although patients undergoing laparoscopic excision had potentially longer hospital stays, this did not necessarily translate into higher costs. The trend toward longer length of stay may be attributed to the fact that the two surgeons who performed the laparoscopic procedures opted to leave an indwelling urinary drainage catheter in place post-operatively, which necessitated inpatient admission. One of the surgeons who performed the procedure laparoscopically used this technique exclusively, while the other performed one laparoscopic and one open procedure. The surgeon who performed both types of procedures (one of each) used the same post-operative management for both patients. Therefore, it is a matter of surgeon preference regarding post-operative management rather than the actual procedure (laparoscopic versus open) that resulted in inpatient admission and longer length of stay. Of note, Turial et al., who published a series of 27 children that had undergone laparoscopic excision of a urachal remnant, utilized bladder decompression for 1 to 3 days postoperativelybased on surgeon preference with equivalent outcomes [5].

We feel that urachal remnants in children can safely be managed with a laparoscopic approach based on our retrospective review. We were very fortunate in that none of our patients experienced a complication. While this is also the case in most studies, Naiditch et al. found a post-operative complication rate of $14.7 \%$ [12]. Because of this and other studies which show that some urachal remnants do spontaneously resolve, there is a question as to whether or not urachal remnants can be managed conservatively [13]. Ideally, a randomized, prospective study with a larger number of subjects should be done to answer this question. However, given the rare occurrence of this entity, it is unlikely that an appropriately powered prospective trial comparing the two procedures can be done.

\section{References}

1. Huang CS, Luo CC, Chao HC, Chen HM, Chu SM (2003) Urachal anomalies in children: experience at one institution. Chang Gung Med J 26: 412-416. 
Citation: Whitehead A, Arthur LG, Prasad R. Laparoscopic vs Open Excision of Urachal Remnants in Children. J Surgery. 2015;2(2): 3.

ISSN: 2332-4139

2. Kurtz M, Masiakos PT (2008) Laparoscopic resection of aurachal remnant. J Pediatr Surg 43: 1753-1754.

3. Choi YJ, Kim JM, Ahn SY, Oh JT, Han SW, et al. (2006) Urachal anomalies in children: a single center experience. Yonsei Med J 47: 782-786.

4. McCollum MO, Macneily AE, Blair GK (2003) Surgical implications of urachal remnants: Presentation and management. J Pediatr Surg 38: 798-803.

5. Turial S, Hueckstaedt T, Schier F, Fahlenkamp D (2007) Laparoscopic treatment of urachal remnants in children. J Urol 177: 1864-1866.

6. Galati V, Donovan B, Ramji F, Campbell J, Kropp BP, et al. (2008) Management of urachal remnants in early childhood. J Urol 180: 1824-1826.

7. Kojima Y, Hayashi Y, Yasui T, Itoh Y, Maruyama T, et al. (2007) Laparoscopic management for urachal cyst in a 9-year-old boy. Int Urol Nephrol 39: 771774.

8. Li Destri G, Schillaci D, Latino R, Castaing M, Scilletta B, et al. (2011) The urachal pathology with umbilical manifestation: overview of laparoscopic technique. J Laparoendosc Adv Surg Tech A 21: 809-814.

9. Okegawa T, Odagane A, Nutahara K, Higashihara E (2006) Laparoscopic management of urachal remnants in adulthood. Int J Urol 13: 1466-1469.

10. Bertozzi M, Riccioni S, Appignani A (2014) Laparoscopic treatment of symptomatic urachal remnants in children. J Endourol 28: 1091-1096.

11. Masuko T, Uchida H, Kawashima H, Tanaka Y, Deie K, et al. (2013) Laparoscopic excision of urachal remnants is a safe and effective alternative to open surgery in children. J Laparoendosc Adv Surg Tech A 23: 1016-1019.

12. Naiditch JA, Radhakrishnan J, Chin AC (2013) Current diagnosis and management of urachal remnants. J Pediatr Surg 48: 2148-2152.

13. Nogueras-Ocaña $M$, Rodríguez-Belmonte R, Uberos-Fernández J, JiménezPacheco A, Merino-Salas S, et al. (2014) Urachal anomalies in children: surgical or conservative treatment? J Pediatr Urol 10: 522-526. 